\title{
Liquid Chromatography Tandem Mass Spectrometry Determination of Bicalutamide in Human Plasma and Application to a Bioequivalence Study
} Bora Kim, JunHwa Shim, SeungHwan Lee, Kyung-Sang Yu, Seo Hyun Yoon and Joo-Youn Cho*

Department of Pharmacology and Clinical Pharmacology, Seoul National University College of Medicine and Hospital, Seoul, Republic of Korea

\begin{abstract}
This study aimed to develop a highly sensitive, rapid method to evaluate the pharmacokinetic bioequivalence of two formulations of the anticancer drug, bicalutamide. According to US and Korean regulatory requirements for a bioequivalence test, we developed an achiral, bioanalytical method to determine bicalutamide levels in human plasma. The method included liquid chromatography tandem mass spectrometry in negative mode and was validated with nilutamide as an internal standard. Quantitation was performed for the transition of $429.2 \rightarrow 255.0$ (m/z) for bicalutamide and $316.2 \rightarrow 273.2(\mathrm{~m} / \mathrm{z})$ for nilutamide. The lower limit of quantitation was $10 \mathrm{ng} \mathrm{mL}^{-1}$ with a $50 \mu \mathrm{L}$ plasma sample. This sensitivity was about 8 times higher than current methods in pharmaceuticals and 10 times higher than methods for human plasma samples. The concentrations of seven working standards showed linearity between 10 and $2000 \mathrm{ng} \mathrm{mL}^{-1}\left(r^{2} \geq 0.9993\right)$. Chromatographic separation was achieved within 4 min, compared to the 10 min of current methods. We used a Luna C18 column $(100 \mathrm{~mm} \times 2 \mathrm{~mm}, 5 \mu \mathrm{m})$ with distilled water/acetonitrile $(30 / 70, v / v)$ as an isocratic mobile phase with a flow rate of $0.3 \mathrm{~mL} \mathrm{~min}^{-1}$. The average extraction recoveries of 3 quality control concentrations were $94.43 \%$ for bicalutamide and $99.28 \%$ for nilutamide. The coefficient of variation was $\leq 15 \%$ for intra- and inter-batch assays. Thus, this method satisfied the US and Korean validation requirements. When applied to a pharmacokinetic bioequivalence study in 33 healthy Korean male volunteers, this method showed high sensitivity, selectivity, and accuracy.
\end{abstract}

Keywords: Validation; LC-MS-MS; Pharmacokinetic bioequivalence study; Bicalutamide

\section{Introduction}

Bicalutamide, N-[4-cyano-3(trifluoromethyl) phenyl]-3-[(4fluorophenyl sulfonyl)]-2-hydroxy-2-methyl propanamide, is an oral, nonsteroidal, anti-androgen agent used for treating prostate cancer. Bicalutamide treatment prevents the activation of the androgen receptor, which inhibits the growth of prostate cancer cells in patients with early, non-metastatic, or advanced prostate cancer [1,2]. Bicalutamide is a racemate that shows anti-androgenic activity in the R-enantiomer form, but little or no anti-androgenic activity in the S-enantiomer form. Several early publications described methods for identifying bicalutamide enantiomers in pharmaceuticals with liquid chromatography with ultraviolet detection (LC-UV) [3-5]. In addition, a previous exploratory study described bicalutamide levels in human plasma with liquid chromatography and tandem mass spectrometry (LC-MS-MS) [6]. The present study was conducted to develop a method for evaluating the pharmacokinetic bioequivalence of two formulations of bicalutamide in clinical trials. Our methods were designed to meet US and Korean regulatory requirements for a bioequivalence test $[7,8]$. The guidance for bioequivalence studies recommends measuring the racemate with an achiral assay. We developed an achiral HPLC-MS-MS method for determining bicalutamide in human plasma. This sensitive, rapid, high throughput method requires only a simple plasma sample preparation. With a small plasma sample $(50 \mu \mathrm{L})$, we quantitated the bicalutamide concentration in the range of $10-2000 \mathrm{ng} \mathrm{ml}^{-1}$. The lower limit of quantitation $\left(10 \mathrm{ng} \mathrm{ml}^{-1}\right)$ was about 8 times more sensitive than the previously published methods in pharmaceuticals and 10 times more sensitive than the methods in human plasma samples [3,6]. With our method, the total run time was reduced to $4 \mathrm{~min}$ from $10 \mathrm{~min}$ in a previous study [3]. In a bioequivalence study, the current method was suitable for comparing the pharmacokinetic profiles of two formulations with high sensitivity, selectivity, and accuracy.

\section{Experimental Methods}

\section{Chemicals and reagents}

Bicalutamide and nilutamide were obtained from Sandoz Korea Ltd. (Seoul, Korea) and Sigma-Aldrich (St. Louis, MO, USA), respectively. Nilutamide was used as an internal standard (IS) for this assay. Acetonitrile (HPLC grade) was purchased from Fisher Scientific, Korea Ltd.

\section{Chromatographic and mass spectrometry conditions}

High performance liquid chromatography (HPLC) was performed on an Agilent (Santa Clara, CA, USA) 1100 series HPLC with a Luna C18 column $(100 \times 2 \mathrm{~mm}, 5 \mu \mathrm{m}$, Phenomenex, USA). The column temperature was set at $25^{\circ} \mathrm{C}$. The mobile phase was distilled water and acetonitrile at a ratio of 30:70, applied at a flow rate of $0.3 \mathrm{~mL} \mathrm{~min}$ 1. Sample analyses were performed on a tandem quadrupole mass spectrometer (MS/MS) API 4000 (Applied Biosystems/MDS Sciex, Toronto, Canada), operated in negative electrospray ionization mode. The MS/MS detection was conducted by multiple reaction monitoring at a transition of $429.2 \rightarrow 255.0(\mathrm{~m} / \mathrm{z})$ for bicalutamide and $316.2 \rightarrow$ $273.2(\mathrm{~m} / \mathrm{z})$ for the IS (Figure 1). For both bicalutamide and the IS, the source-dependent parameters were as follows: Gas 1 (nebulizer gas): 40

*Corresponding author: Joo-Youn Cho, Department of Pharmacology and Clinical Pharmacology, Seoul National University College of Medicine and Hospital, 101 Daehangno, Jongno-gu, Seoul, Republic of Korea, 110-744, Tel: +82-2-740-8286; Fax: +82-2-742-9252; E-mail: joocho@snu.ac.kr

Received June 27, 2011; Accepted July 21, 2011; Published July 27, 2011

Citation: Kim B, Shim J, Lee S, Yu K, Hyun S, et al. (2011) Liquid Chromatography Tandem Mass Spectrometry Determination of Bicalutamide in Human Plasma and Application to a Bioequivalence Study. J Bioanal Biomed 3: 098-102. doi:10.4172/1948-593X.1000051

Copyright: (c) $2011 \mathrm{Kim} \mathrm{B}$, et al. This is an open-access article distributed under the terms of the Creative Commons Attribution License, which permits unrestricted use, distribution, and reproduction in any medium, provided the original author and source are credited. 
Citation: Kim B, Shim J, Lee S, Yu K, Hyun S, et al. (2011) Liquid Chromatography Tandem Mass Spectrometry Determination of Bicalutamide in Human Plasma and Application to a Bioequivalence Study. J Bioanal Biomed 3: 098-102. doi:10.4172/1948-593X.1000051

psig; Gas 2 (heater gas flow): 50 psig; ion spray voltage: $4500 \mathrm{~V}$; turbo heater temperature: $400^{\circ} \mathrm{C}$; interface heater: $\mathrm{On}$; entrance potential: 10 $\mathrm{V}$; collision activation dissociation gas: 6 psig; and curtain gas, nitrogen: 10 psig. The optimized values for analyte-dependent parameters were set as follows: declustering potential, collision energy, and cell exit potential were set to: 75,22 , and $9 \mathrm{~V}$, respectively, for bicalutamide and 90,30 , and $9 \mathrm{~V}$, respectively, for the IS. Quadrupoles 1 and 3 were maintained at unit mass resolution and the dwell time was set at 400 ms.

\section{Standard and quality control (qc) sample preparation}

Bicalutamide and the IS stock solutions were prepared in methanol to a final concentration of $1 \mathrm{mg} \mathrm{mL}^{-1}$. The appropriate dilutions with acetonitrile were performed to produce 7 working standards of 100,50 , $25,5,2.5,1$, and $0.5 \mu \mathrm{g} \mathrm{mL}^{-1}$ of bicalutamide and one $50 \mathrm{ng} \mathrm{mL}^{-1}$ sample of IS. Calibration standards ranging from 10 to $2000 \mathrm{ng} \mathrm{mL}^{-1}$ were prepared by spiking $20 \mu \mathrm{L}$ of the working standards into $1 \mathrm{~mL}$ of blank plasma. The QC samples were prepared with a different bicalutamide working solution and diluted in blank plasma. The QC bicalutamide concentrations included $10 \mathrm{ng} \mathrm{mL}^{-1}$ for the lower limit of quantitation (LLOQ), $20 \mathrm{ng} \mathrm{mL}^{-1}$ for a low quality control (LoQC), $200 \mathrm{ng} \mathrm{mL}^{-1}$ for a Middle quality control (MeQC), $1600 \mathrm{ng} \mathrm{mL}^{-1}$ for a High quality control (HiQC), and $4000 \mathrm{ng} \mathrm{mL}^{-1}$ for a Di quality control (DiQC). All diluted samples were stored at $-70^{\circ} \mathrm{C}$.

\section{Sample preparation}

Frozen human plasma was thawed at room temperature. A plasma sample $(50 \mu \mathrm{L})$ was placed in a $1.5 \mathrm{~mL}$ polypropylene tube, and $950 \mu \mathrm{L}$ of IS ( $50 \mathrm{ng} \mathrm{mL}^{-1}$ ) or one of the bicalutamide solutions was added. After vortexing for $30 \mathrm{~s}$ and centrifuging at 14,000 rpm for $5 \mathrm{~min}, 50 \mu \mathrm{L}$ of supernatant was transferred to another polypropylene tube, and $950 \mu \mathrm{L}$

(a)

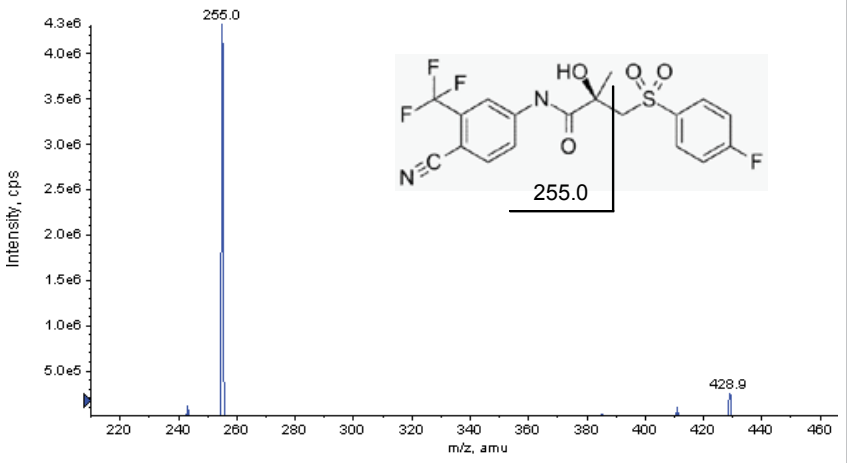

(b)

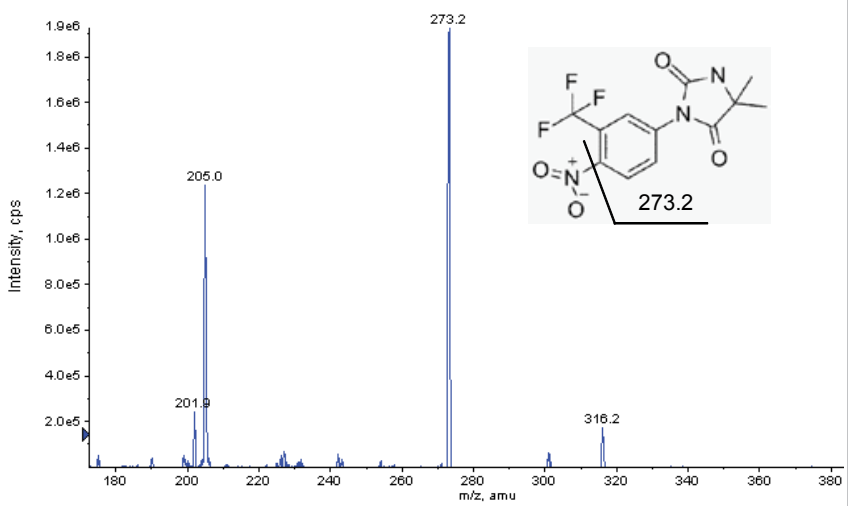

Figure 1: Product ion spectra of (a) bicalutamide $(255.0 \mathrm{~m} / \mathrm{z})$ and (b) nilutamide $(273.2 \mathrm{~m} / \mathrm{z})$; the chemical structure of each compound is shown in the inset. of $70 \%$ acetonitrile (mobile phase) was added. After vortexing for $30 \mathrm{~s}$, $4 \mu \mathrm{L}$ of each sample was injected into the LC-MS/MS system.

\section{Bioanalytical Method Validation}

Validation was performed according to the FDA guidelines for Bioanalytical Methods Validation for Human Studies [7,8].

\section{Linearity and sensitivity}

To test for acceptable linearity, 7 calibration standards were prepared, and more than 5 standards had to be within $\pm 15 \%$ of the mean calculated concentrations. Calibration curves were calculated with weighted $(1 / \mathrm{x})$ linear regression analyses and fitted with regression coefficients of $>0.99$. To test for sensitivity, the LLOQ of $10 \mathrm{ng} \mathrm{mL}^{-1}$ was allowed a deviation of $\pm 20 \%$.

\section{Accuracy and precision}

Intra-batch precision and accuracy were evaluated with 6 replicates of QC samples at each concentration in a single assay on 5 different days. Intra- and inter-batch precisions were expressed as coefficients of variation (CV\%). For validation, the CV had to be within $\pm 15 \%$, except for the LLOQ, in which the deviation could not exceed $\pm 20 \%$. The accuracy was calculated as the percent deviation from the nominal concentration, and more than 5 replicates had to be within $\pm 20 \%$.

\section{Extraction recovery}

In order to evaluate the extraction recovery, we compared QC samples before and after extraction in 6 replicate samples. Before and after extraction, 3 different bicalutamide concentrations $(20,200,1600$

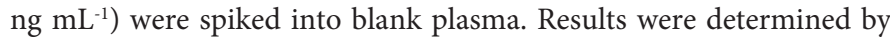
comparing the analyte peak areas.

\section{Selectivity and specificity}

To determine whether there was any interference from endogenous plasma constituents, we analyzed 6 blank plasma samples and LLOQ samples made with the same blank plasma. Interference was considered acceptable only when the peak area of the interference did not exceed $20 \%(\mathrm{~S} / \mathrm{N}$ ratio $=5)$ of the peak area of the LLOQ.

\section{Stability}

The stability of the stock solution was validated after placing the bicalutamide stock solution at room temperature for $41 \mathrm{~h}$. The concentration was compared with that of a freshly prepared stock solution and the stability was expressed as the percent difference; for validation, the difference could not exceed $\pm 10 \%$. The freeze/thaw stability was determined by comparing a back-calculated concentration of QCs after 3 freeze/thaw cycles to QCs after 1 freeze/thaw cycle. Extraction stability was also tested because it required time to inject the sample into the HPLC with the autosampler. To test extraction stability, each of 6 extracted samples from LLOQ to DiQC were stored at $4^{\circ} \mathrm{C}$ in the autosampler for $25 \mathrm{~h}$. These were compared to freshly extracted QC samples, and the concentration was expressed as a percentage of the freshly extracted QC concentration. To validate whether the analyte was stable in the plasma, we performed short-term stability by storing LoQC and HiQC samples at room temperature for $24 \mathrm{~h}$ and comparing them to freshly prepared QCs.

\section{Application to a bioequivalence study}

The validated analytical assay was applied to a pharmacokinetic bioequivalence study. A two-period, crossover design study was conducted with 34 healthy Korean male volunteers. The mean (SD) 
Citation: Kim B, Shim J, Lee S, Yu K, Hyun S, et al. (2011) Liquid Chromatography Tandem Mass Spectrometry Determination of Bicalutamide in Human Plasma and Application to a Bioequivalence Study. J Bioanal Biomed 3: 098-102. doi:10.4172/1948-593X.1000051

age, height, and weight of subjects were $25.8(4.1)$ years, $173.6(5.7) \mathrm{cm}$, and 68.9 (7.8) kg, respectively. The clinical protocol was reviewed and approved by the institutional review board of Seoul National University Hospital (SNUH) and the Korean Food and Drug Administration. All subjects provided informed consent to participate in the study. In the first period, subjects randomly received a single dose of bicalutamide in either the test formulation (30 mg tablet, Cadexo, Sandoz Korea Ltd., Seoul, Korea) or the reference formulation (Casodex, AstraZeneca UK Ltd, Macclesfield, United Kingdom). The first period was followed by an interval of $\mathrm{x}$ days before the second period began. In the second (a)

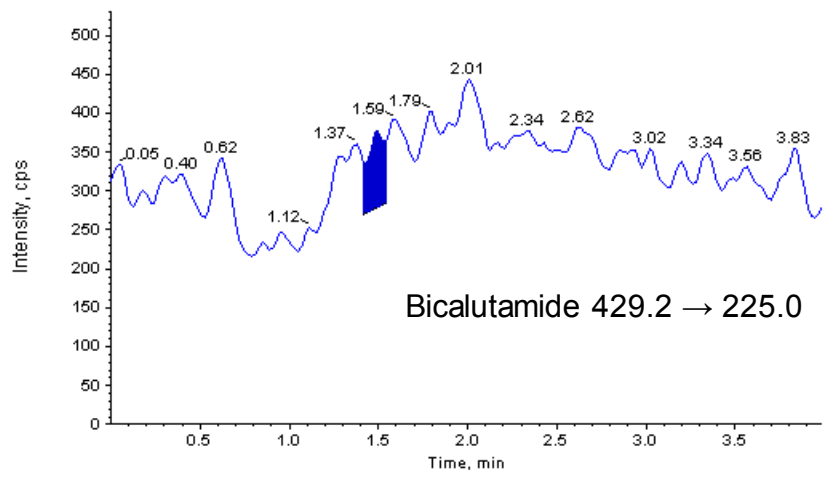

(b)

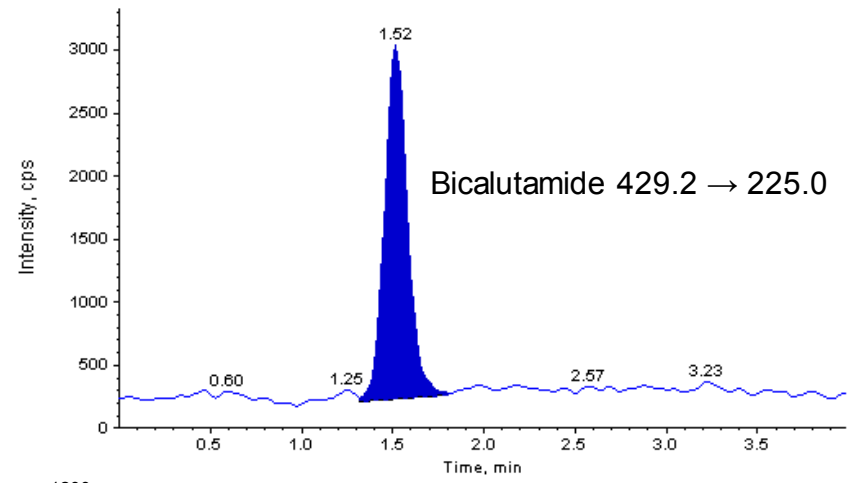

(c)
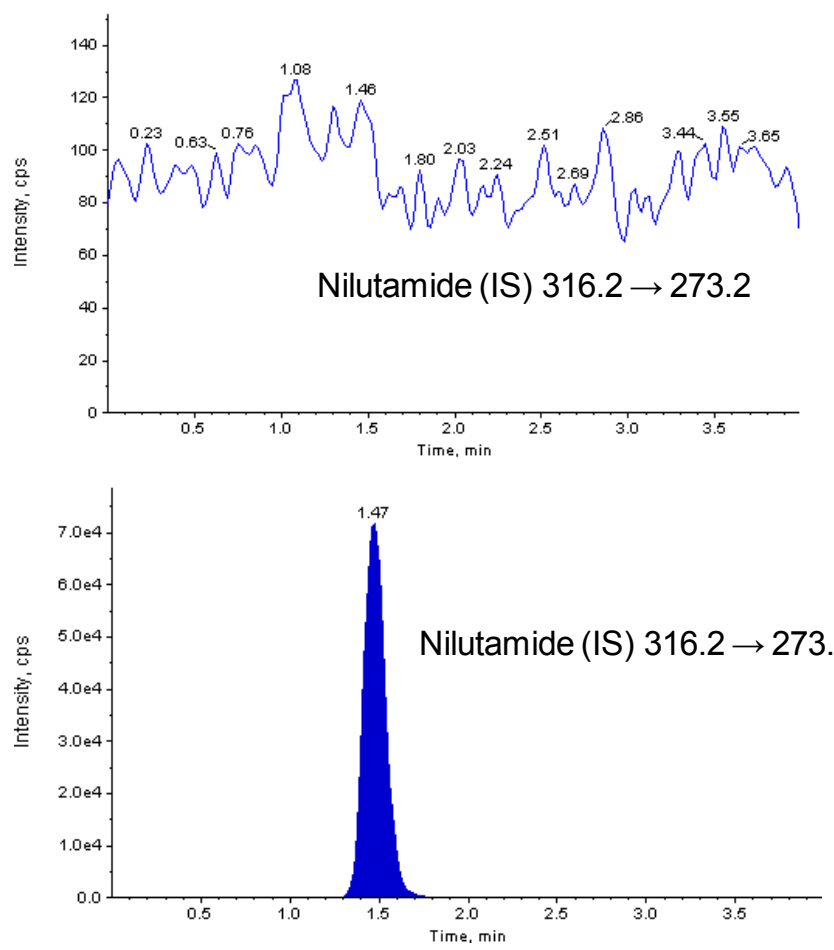

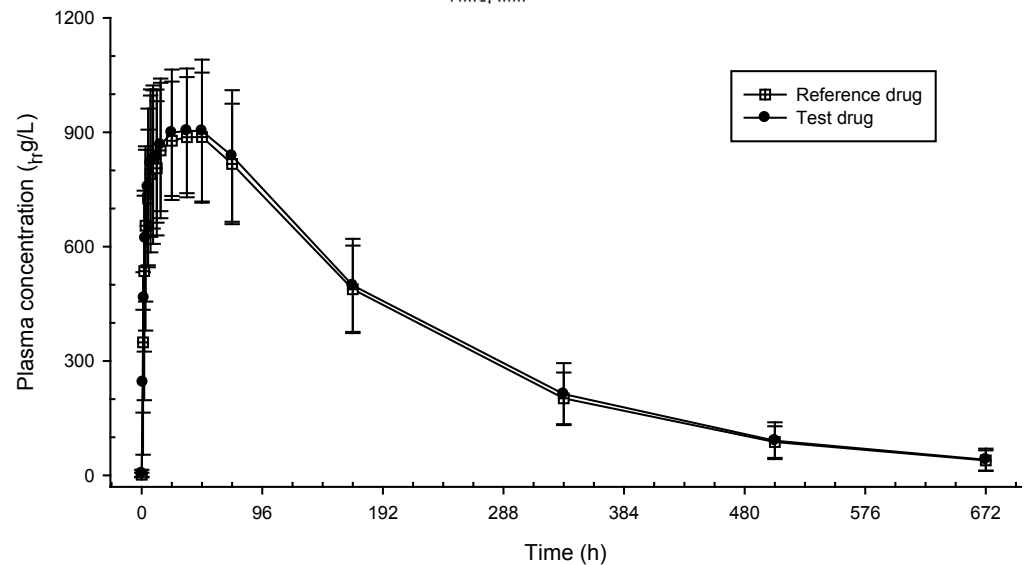

Figure 2 Multiple reaction monitoring ion chromatograms of bicalutamide $(429.2 \rightarrow 225.0$ ) and nilutamide (IS); (a) blank plasma; (b) LLOQ sample (10 ng/ml); (c) mean (SD) plasma bicalutamide concentrations over time after administration of a single oral dose of either the reference formulation (squares) or test formulation (circles) of bicalutamide $(50 \mathrm{mg})(\mathrm{n}=33)$

\begin{tabular}{|c|c|c|c|c|c|c|c|c|c|}
\hline \multirow{2}{*}{ QC } & \multirow{2}{*}{$\begin{array}{r}\text { Concentration } \\
\text { added }\left(\mathrm{ng} \mathrm{mL}^{-1}\right)\end{array}$} & \multicolumn{4}{|c|}{ Intra-batch $(n=6)$} & \multicolumn{4}{|c|}{ Inter-batch $(n=6)$} \\
\hline & & Mean $\left(\mathrm{ng} \mathrm{mL}^{-1}\right)$ & $\mathrm{SD}( \pm)$ & Precision (\%CV) & Accuracy (\%) & Mean $\left(\mathrm{ng} \mathrm{mL}^{-1}\right)$ & $\mathrm{SD}( \pm)$ & Precision (\%CV) & Accuracy (\%) \\
\hline LLoQ & 10 & 10.36 & 0.25 & 2.39 & 103.60 & 10.24 & 0.44 & 6.48 & 102.40 \\
\hline LoQC & 20 & 19.72 & 0.98 & 4.98 & 98.59 & 19.87 & 0.97 & 3.60 & 99.37 \\
\hline MeQC & 200 & 200.40 & 7.47 & 3.73 & 100.20 & 196.70 & 3.26 & 3.15 & 98.36 \\
\hline HiQC & 1600 & 1657.00 & 89.54 & 5.41 & 103.60 & 1627.00 & 45.15 & 2.29 & 101.70 \\
\hline DiQC & 4000 & 3918.00 & 58.37 & 1.49 & 97.95 & & & & \\
\hline
\end{tabular}

*DiQC was analyzed by 10 -fold dilution

Table 1: Intra- and inter-day precision and accuracy for bicalutamide in human plasma. 
Citation: Kim B, Shim J, Lee S, Yu K, Hyun S, et al. (2011) Liquid Chromatography Tandem Mass Spectrometry Determination of Bicalutamide in Human Plasma and Application to a Bioequivalence Study. J Bioanal Biomed 3: 098-102. doi:10.4172/1948-593X.1000051

\begin{tabular}{|c|c|c|c|}
\hline Sample concentration $\left(\mathrm{ng} \mathrm{mL}^{-1}\right)$ & Mean concentration $\left(\mathrm{ng} \mathrm{mL}^{-1}\right) \pm \mathrm{SD}(\%)(\mathrm{n}=6)$ & Precision (\%CV) & Accuracy (\%) \\
\hline \multicolumn{4}{|l|}{ Extract stability $\left(4^{\circ} \mathrm{C}\right.$ for $\left.25 \mathrm{~h}\right)$} \\
\hline 10 & $11.29 \pm 0.98$ & 8.69 & 112.93 \\
\hline 20 & $21.09 \pm 2.03$ & 9.62 & 105.43 \\
\hline 200 & $195.42 \pm 3.16$ & 1.62 & 97.71 \\
\hline 1600 & $1650.33 \pm 104.41$ & 6.33 & 103.15 \\
\hline 4000 & $3878.67 \pm 68.54$ & 1.77 & 96.97 \\
\hline \multicolumn{4}{|l|}{ Freeze/thaw stability ${ }^{a}$} \\
\hline 20 & $21.25 \pm 2.10$ & 9.86 & 106.23 \\
\hline 1600 & $1547 \pm 12$ & 0.79 & 96.7 \\
\hline \multicolumn{4}{|c|}{ Short-term stability (room temperature for $24 \mathrm{~h}$ ) } \\
\hline 20 & $18.34 \pm 0.44$ & 2.42 & 91.71 \\
\hline 1600 & $1685 \pm 13.52$ & 0.8 & 105.33 \\
\hline
\end{tabular}

${ }^{a}$ Freeze thaw stability after third cycle

Table 2: Stability data for bicalutamide in human plasma.

period, subjects received a single dose of the alternate bicalutamide formulation (that not received in the first period). Serial blood samples were obtained before dosing and at $1,2,3,5,7,9,12,15,24,36,48,72$, $168,336,504$, and $672 \mathrm{~h}$ after each administration. Pharmacokinetic analyses were performed with the noncompartmental method of Win-Nolin, version 5.2.1 (Pharsight Corporation, Mountain View, California). Maximum plasma concentration $\left(\mathrm{C}_{\mathrm{max}}\right)$ and the time point of maximum plasma concentration were obtained directly by individual plasma concentration-time profiles. The terminal elimination constant $\left(\lambda_{z}\right)$ was estimated with linear regression, and the elimination half-life $\left(\mathrm{t}_{1 / 2}\right)$ was calculated as $\ln 2 / \lambda_{\mathrm{z}}$. For $\mathrm{AUC}_{0-672 \mathrm{~h}}$ and $\mathrm{AUC}_{0-\infty}$, we used the linear-up/log-down trapezoidal method.

\section{Results and Discussion}

\section{Method development}

Determinations of bicalutamide and the IS were optimized with HPLC-MS/MS. The mass spectrometer was tuned in negative ionization mode, with deprotonated ions $[\mathrm{M}-\mathrm{H}]$. For each selected multiple reaction monitoring transition of bicalutamide $(\mathrm{m} / \mathrm{z} 429.2 \rightarrow$ $255.0)$ and IS $(\mathrm{m} / \mathrm{z} 316.2 \rightarrow 273.2)$, retention times were $1.42 \mathrm{~min}$ and $1.47 \mathrm{~min}$, respectively. The total run time was $4 \mathrm{~min}$ (Figure $2 \mathrm{a}$ and Figure 2b).

\section{Validation of the Analytical Method}

\section{Linearity and sensitivity}

The 7-point calibration curve was reproducible and linear over the range of $10-2000 \mathrm{ng} \mathrm{mL}^{-1}$. The calibration curve showed good linearity for bicalutamide. The regression equation was $\mathrm{y}=0.154 \mathrm{x}+0.00535$, and the correlation coefficient $(r)$ was over 0.9993 . The LLOQ was found to be $10 \mathrm{ng} \mathrm{mL}^{-1}$, which gave a signal to noise ratio of $5(\mathrm{~S} / \mathrm{N} \geq 5)$.

\section{Accuracy and precision}

For the intra-batch assay, the precision ranged from $1.49 \%$ to $5.41 \%$, and the accuracy ranged from $97.95 \%$ to $103.6 \%$. Inter-batch precision and accuracy ranged from $2.29 \%$ to $6.48 \%$ and $98.36 \%$ to $102.4 \%$, respectively (Table 1). These data indicated that the method had sufficient accuracy and precision for determining bicalutamide concentrations.

\section{Extraction recovery}

The pre-extraction, spiked QC samples were compared to postextraction samples. For each of the 3 concentrations tested, the extraction recoveries were $98.91 \%, 89.88 \%$, and $94.50 \%$. The mean extraction recovery was $94.43 \pm 4.52 \%$. The recovery of nilutamide (IS) was $99.28 \% \pm 4.30 \%$.

\section{Selectivity and specificity}

We tested 6 blank plasma samples from different subjects. All 6 blank plasma samples had endogenous peaks at the retention time of bicalutamide. However, the peak area was within $9.781 \%$ of the peak area of the LLOQ (10 $\mathrm{ng} \mathrm{mL}^{-1}$ ) (Figure $2 \mathrm{a}$ and Figure $2 \mathrm{~b}$ ). Thus, the interference peak in blank plasma was not considered to be specific to bicalutamide.

\section{Stability}

The stock solution stored for $41 \mathrm{~h}$ at room temperature showed a $-9.51 \%$ difference in concentration compared to that of freshly prepared stock solution. To be considered stable, the permissible percent difference from freshly prepared solution was $\pm 15 \%$ for extract stability, freeze/thaw stability, and short-term stability. We found that the extract stability of QC samples stored at $4^{\circ} \mathrm{C}$ for $25 \mathrm{~h}$ was within the permissible range $( \pm 15 \%)$. The freeze/thaw stability did not show any relevant difference between QC samples that underwent 1 or 3 freeze/ thaw cycles. The short-term stability of bicalutamide was stable at room temperature for $24 \mathrm{~h}$ (Table 2).

\section{Application}

The assay was applied in a clinical trial to determine the plasma concentration after an oral administration of bicalutamide in 33 healthy Korean male volunteers. The mean (SD) plasma concentration change over time is shown in Figure 2c. Concentrations were measured after administration of a single oral dose of one of two formulations of bicalutamide 50-mg tablets. The geometric mean ratio of the test formulation to the reference formulation showed a $90 \% \mathrm{CI}$ within 0.97 to 1.06 for $\mathrm{C}_{\max }$ and 0.98 to 1.07 for $\mathrm{AUC}_{0-672 \mathrm{~h}}$. The results indicated that there were no significant differences between the reference and test formulations [2].

\section{Conclusion}

Although an HPLC assay for bicalutamide enantiomers in pure and pharmaceutical dosage forms was published previously, we developed a highly sensitive, rapid, accurate method that was successfully applied to a bioequivalence study. Our method was based on LC-ESI-MS/MS analysis of a simple plasma sample preparation. The validation showed that this assay had a high sensitivity with a LLOQ at $10 \mathrm{ng} \mathrm{mL}^{-1}$ and only required a small volume of plasma $(50 \mu \mathrm{L})$. This represented an advantage over the published method, which showed a $0.1 \mu \mathrm{g} \mathrm{mL}^{-1}$ of 
Citation: Kim B, Shim J, Lee S, Yu K, Hyun S, et al. (2011) Liquid Chromatography Tandem Mass Spectrometry Determination of Bicalutamide in Human Plasma and Application to a Bioequivalence Study. J Bioanal Biomed 3: 098-102. doi:10.4172/1948-593X.1000051

LLOQ for each of the bicalutamide enantiomers [6]. In addition, our method required a total run time of $4 \mathrm{~min}$, which was faster than the published $10 \mathrm{~min}$ required for other assays. Our method also showed good linearity in the dynamic range of $10-2000 \mathrm{ng} \mathrm{mL}^{-1}$. These results provided validation for the method. This method was successfully applied in a human clinical study to compare the pharmacokinetic profiles of two formulations of bicalutamide in 33 healthy Korean male volunteers.

\section{Acknowledgments}

This study was supported by Sandoz Korea Ltd., Seoul, Korea

\section{References}

1. Furr BJ (1996) The development of Casodex (bicalutamide): preclinical studies Eur Urol 29 Suppl 2: 83-95

2. Lee S, Chung YJ, Kim BH, Shim JH, Yoon SH, et al. (2009) Comparative pharmacokinetic evaluation of two formulations of bicalutamide 50-mg tablets: an open-label, randomized-sequence, single-dose, two-period crossover study in healthy Korean male volunteers. Clin Ther 31: 3000-3008.
3. Nageswara Rao R, Narasa Raju A, Nagaraju D (2006) An improved and validated LC method for resolution of bicalutamide enantiomers using amylose tris-(3,5-dimethylphenylcarbamate) as a chiral stationary phase. J Pharm Biomed Anal 42: 347-353.

4. Lakshmana RA, Rao JV (2009) RASAYAN J Chem 2: 512-515

5. McKillop D, Boyle GW, Cockshott ID, Jones DC, Phillips PJ, et al. (1993) Metabolism and enantioselective pharmacokinetics of Casodex in man. Xenobiotica 23: 1241-1253.

6. Boccardo F, Rubagotti A, Conti G, Potenzoni D, Manganelli A, et al. (2005) Exploratory study of drug plasma levels during bicalutamide $150 \mathrm{mg}$ therapy co-administered with tamoxifen or anastrozole for prophylaxis of gynecomastia and breast pain in men with prostate cancer. Cancer Chemother Pharmacol 56: 415-420.

7. US Food and Drug Administration (2009) Guidance for Industry. Bioavailability and bioequivalence studies for orally administered drug products-general considerations.

8. Korea Food and Drug Administration (2009) Guidance document for bioequivalence studies 2008 [inKorean] 